\title{
Improving the Health of Teeth in Cats and Dogs with Live Probiotic Bacteria
}

\author{
Veli-Matti Mäkinen1, Annika Mäyrä1, Eveliina Munukka2 ${ }^{2}$ \\ ${ }^{1}$ Gutguide Ltd., Halikko, Finland \\ ${ }^{2}$ Medical Faculty, Turku University, Turku, Finland \\ Email: annika.mayra@versofinland.fi
}

How to cite this paper: Mäkinen, V.-M., Mäyrä, A. and Munukka, E. (2019) Improving the Health of Teeth in Cats and Dogs with Live Probiotic Bacteria. Journal of Cosmetics, Dermatological Sciences and Applications, 9, 275-283.

https://doi.org/10.4236/jcdsa.2019.94024

Received: August 22, 2019

Accepted: October 19, 2019

Published: October 22, 2019

Copyright (C) 2019 by author(s) and Scientific Research Publishing Inc. This work is licensed under the Creative Commons Attribution International License (CC BY 4.0).

http://creativecommons.org/licenses/by/4.0/

\begin{abstract}
Background: Periodontal infections are the most common disease of cats and dogs. About $70 \%-80 \%$ of them suffer from these infections during their lifetime. These infections occur in two forms, gingivitis and periodontitis. Gingivitis is a reversible inflammation of the gingiva which can be cured. As with humans, the harmful bacteria can cause infections also in organs such as the heart, liver and kidneys. Besides veterinary treatment, also the owner's everyday care is essential. Objective: The aim of the study was to develop and clinically test easy home care probiotic product for cats' and dogs' oral health. Methods: Nine cats and thirteen dogs were given a combination of selected bacteria Streptococcus thermophilus SP4, Lactobacillus plantarum 14D and Lactobacillus rhamnosus SP1 (Petguide powder) in powder form daily for four weeks on top of the dry feed. Microbiological samples were taken before and after the intervention from each dog and cat. The samples were analyzed with flow cytometric analysis using Porphyromonas gingivalis and Fusobacterium $s p$. as indicators for oral health. Results: The home care treatment with these specific, live probiotic bacteria was shown to prevent the growth of infectious bacteria within four weeks. Petguide probiotic powder reduced the relative abundance of infectious microbes significantly $(p=0.005)$ in cats and dogs. Conclusion: The results suggest that the probiotic product with three live strains of probiotic bacteria prevents the growth of infectious bacterial species that are known to cause infections in pets' mouths. Therefore, this probiotic combination could be used to improve the oral health of pets and increase their quality of life.
\end{abstract}

\section{Keywords}

Oral Health, Cats and Dogs, Oral Probiotics, Pets, Petguide LAB Powder, Gingivitis, Periodontitis, Probiotic Bacteria, Streptococcus thermophilus SP4, 


\section{Introduction}

The bad breath of cats and dogs has not been considered a symptom of gingivitis or periodontitis until recently. Unfortunately, bad breath may often be the only symptom for the beginning of a serious periodontal disease. Periodontal disease has two stages: gingivitis and periodontitis [1]. In the early stages of gingivitis, the gums become swollen, red and may bleed. In its more serious form, periodontitis, the gums are inflamed, bone can be lost, and the teeth may loosen or fall out [2]. The primary cause of gingivitis is poor or ineffective oral hygiene, which leads to the accumulation of dental plaque at the gum line. In the plaque, the number of microbial cells is high $\left(10^{11} \mathrm{cfu} / \mathrm{g}\right)$ and diversity is rich, containing hundreds of different species [3].

Periodontal disease is a common disease found in dogs and its prevalence increases with age. Small dogs, such as toy and miniature breeds, are more severely affected [4]. The prevalence rates of gingivitis and periodontitis in dogs have been reported to range from $95 \%-100 \%$ and $50 \%-70 \%$, respectively [5]. Up to $80 \%$ of all dogs are believed to have some degree of periodontal disease by the age of two [6].

Only limited information regarding the oral microbiota in dogs has been published [7]. Recent research has established that the bacteria associated with gum disease in dogs are not the same as in humans [3]. However, in another study, it was shown that the species in dogs and their owners were partly the same [8]. It is not certain what species are most responsible for causing gum disease, but it has been suggested that four species of gram-negative anaerobic species are the most common, Actinobacillus actinomycetemcomitans, Porphyromonas gingivalis, Bacteroides forsythus and Eikenella corrodens [9]. However, Tannerella forsythia and Campylobacter rectus, which are known as human periodontitis related species, have been detected with high frequency in dogs [10] and T. forsythia in cats [11]. In a study by Kato et al. (2011), Porphyromonas gulae was analysed as the major species detected in dogs.

In humans, the common species causing gingivitis are Porphyromonas gingivalis and Fusobacteria sp. The oral microbiota contains hundreds of species both in humans and pets, and partly overlapping bacterial groups and species have been detected. The selection of bacterial species in pets seems to depend on breed, feed and domestic teeth care.

The prevention of periodontal diseases has been studied, and the most common treatment is scaling [12]. However, scaling only removes the existing calculi for a short time and calculi starts to form again soon after the procedure. Scaling is done by a veterinarian under anaesthesia, which always poses a risk and burden, especially for ageing pets. The most preventive cure is tooth brushing, but 
as it is a demanding and time-consuming treatment both for the owner and the pet, it is not widely used. Also, types of toothpaste that actively support one of the innate defence mechanisms in saliva, the lactoperoxidase system, have been developed [13].

The oral microbiota in humans is well documented from the first days after birth. The dominant bacterial species being Streptococcus salivarius, which can colonise the mouth [14]. Selected $S$. salivarius strains are used as oral probiotics, which have been shown to prevent the growth of gingivitis and periodontitis causing bacteria [15] [16] as well as yeasts in the mouth [17]. The interest in infectious mouth microbiota is increasing since the recent research results have been connecting them not only to cardiovascular diseases but also for example to the risk of intestinal disorders and Alzheimer's disease [18].

\section{Methods}

In the current study, the effectiveness of specific, viable lactic acid bacteria was studied in the treatment of teeth care of well-kept domestic cats and dogs. Lactic acid bacteria used were Streptococcus thermophilus SP4, Lactobacillus plantarum $14 \mathrm{D}$ and Lactobacillus rhamnosus SP1 (Sacco S, r. 1. Italy) in a ready-mixed powder (later PetGuide LAB powder). The strains were selected based on their known features (survival in oral conditions) and traditional use in food fermentations which makes them safe for owners and pets.

Pure freeze-dried cultures were mixed in microcrystalline cellulose which attaches well on the gums when in contact with saliva. The bacterial level was $10^{9}$ $\mathrm{cfu} / \mathrm{g}$ for each strain. The viability of the strains in the PetGuide product was analysed by flow-cytometry during 12 months storing at room temperature [19].

In the study, the cats and dogs of different ages were fed the PetGuide LAB powder daily by dosing one teaspoon of the powder on top of the dry feed. The actual number of bacteria per animal per day was not the same since several dogs and cats ate from the same cup. The intervention lasted 30 - 40 days. Cats (nine individuals from Exotic and Persian breeds) and 13 dogs of Pomeranian and Peruvian hairless breeds were chosen for the intervention. Six of the cats had healthy teeth and fresh breath, and three had minor plaque formation and bad breath. Of the 13 dogs, seven had bad breath and plaque, most being Peruvian hairless dogs (Table 1 and Table 2).

Microbiological samples were taken with a cotton swab from the upper eye tooth and gum before and after the intervention from each dog and cat. The samples were analysed with specific flow cytometric analysis [20] by enumerating the species of Porphyromonas gingivalis and Fusobacterium sp. which were selected as indicators for oral health. In the Fusobacterium sp.-group several related species included in this group were detected. These species were found both in cats and dogs in easily detectable numbers similarly to human saliva.

Statistical analysis was performed with the Mann Whitney U-test (significance in $\mathrm{p}$-value $<0.05$ ). 
Table 1. Teeth/mouth health and age of the nine cats before the PetGuide LAB study (estimated by owner/veterinarian).

\begin{tabular}{lcc}
\hline CAT BREED & AGE (YEARS) & TEETH/MOUTH HEALTH \\
\hline 1. Exotic & 1.5 & Normal \\
2. Persian & 4 & Some plaque, bad breath \\
3. Exotic & $6 \mathrm{mo}$ & Normal \\
4. Exotic & 2 & Some plaque \\
5. Abessinian & $10 \mathrm{mo}$ & Normal \\
6. Exotic & 4 & Some plaque, bad breath \\
7. Persian & 6 & Some plaque, bad breath \\
8. Exotic & 2.5 & Normal \\
9. Exotic & 2.5 &
\end{tabular}

Table 2. Teeth/mouth health and age of 13 dogs before PetGuide LAB study (estimated by owner/veterinarian).

\begin{tabular}{|c|c|c|}
\hline DOG BREED & AGE (YEARS) & TEETH/MOUTH HEALTH \\
\hline 1. Pomeranian & 1.5 & Normal, no plague \\
\hline 2. Pomeranian & 2 & Normal \\
\hline 3. Pomeranian & 5.5 & Normal, some plaque \\
\hline 4. Pomeranian & 6 & Bad breath, periodontium \\
\hline 5. Pomeranian & 4.5 & Normal \\
\hline 6. Pomeranian & 4.5 & Some plaque \\
\hline 7. Pomeranian & 1 & Normal \\
\hline 8. Pomeranian & 7 & Bad breath, periodontium \\
\hline 9. Peruvian Hairless & 9 & Lots of plaque, bad breath \\
\hline 10. Peruvian Hairless & 2 & Lots of plaque, bad breath \\
\hline 11. Peruvian Hairless & 2 & Lots of plaque, bad breath \\
\hline 12. Peruvian Hairless & 3 & Lots of plaque, bad breath \\
\hline 13. Peruvian Hairless & 4 & Lots of plaque, bad breath \\
\hline
\end{tabular}

\section{Results}

In the cat group $(\mathrm{n}=9)$, feeding with PetGuide powder for four weeks reduced the relative abundance (\% of total microbiota) of $P$. gingivalis species significantly from 0.9 to 0.5 ( $\mathrm{p}=0.028)$. In addition, the amount of Fusobacterium $s p$. was reduced from $2.0 \%$ to $0.9 \%$ but not statistically significantly $(\mathrm{p}=0.075)$ (Figure 1).

In the dog group $(\mathrm{n}=13)$, a significant reduction from 1.5 to 1.1 in the relative abundance of periodontopathic bacteria was seen especially in the amount of Fusobacterium sp. $(\mathrm{p}=0.05$, Figure 2). A trend for reduction in $P$. gingivalis relative abundance was noticed $(\mathrm{p}=0.071$, Figure 2$)$. 


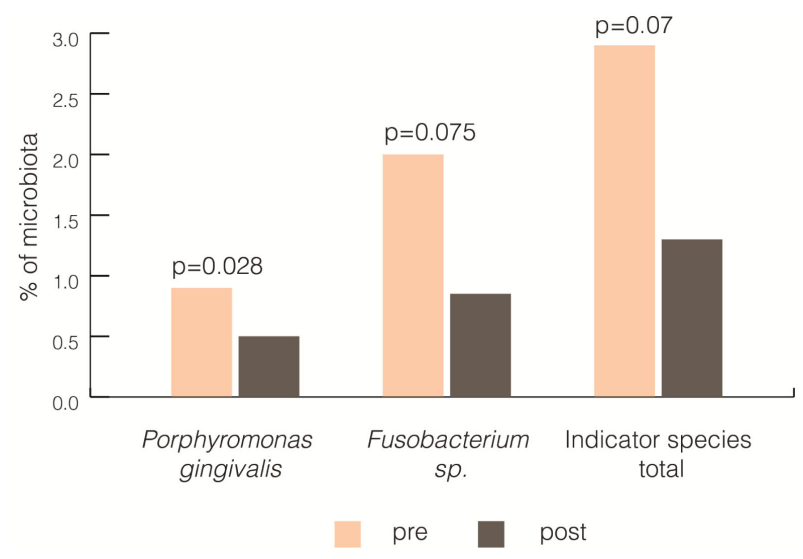

Figure 1. PetGuide treatment for four weeks showed a significant decrease in P. gingivalis and a clear trend in Fusobacterium sp. and in infectious bacteria overall in cats $(\mathrm{n}=9)$.

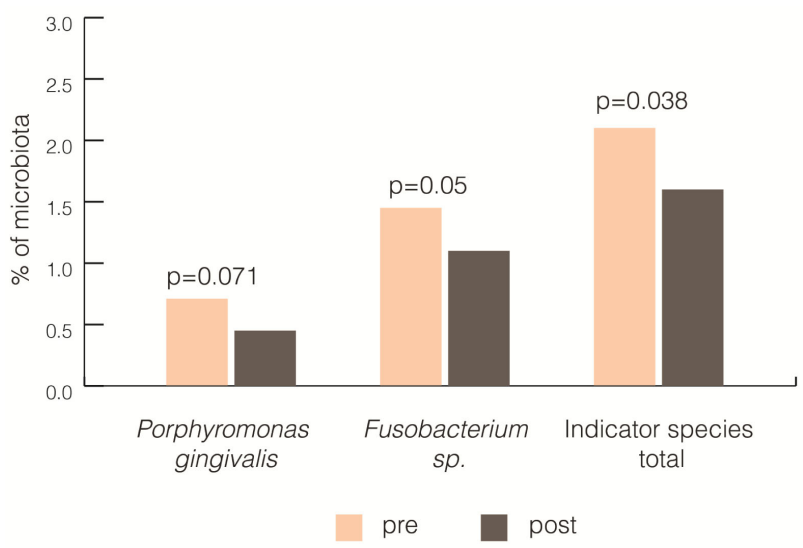

Figure 2. In dogs PetGuide treatment for four weeks showed a significant decrease in Fusobacterium sp. and infectious species overall as well as a decreasing trend in P. gingivalis $(\mathrm{n}=13)$.

A separate analysis of Peruvian hairless dogs with several mouth health problems showed a significant reduction from $1.5 \%$ to $0.6 \%(\mathrm{p}=0.042)$ in Fusobacterium $s p$. and from $2.3 \%$ to $1.2 \%(\mathrm{p}=0.043)$ in infectious bacteria overall (Figure 3). One specific dog with the worst oral health situation showed the most effective reduction both in P. gingivalis (from $1.4 \%$ to $0.5 \%$ ) and Fusobacterium sp. (from $3.2 \%$ to $0.7 \%$, Figure 4 ). The bacterial load was also higher with this dog before the intervention.

Overall, the daily treatment with the PetGuide powder for four weeks reduced the relative abundance of the summarised gum infectious microbes significantly from $2.1 \%$ to $1.4 \%(\mathrm{p}=0.005)$ in cats and dogs (Figure 5$)$.

\section{Discussion}

Gingivitis can be cured with medical treatment, whereas periodontitis will cause stable damages to the tooth tissues, and the damages are often painful to the pets. Therefore the daily domestic oral care by the pet owner is essential in the prevention of severe infections. The lack of preventive care often leads to these 


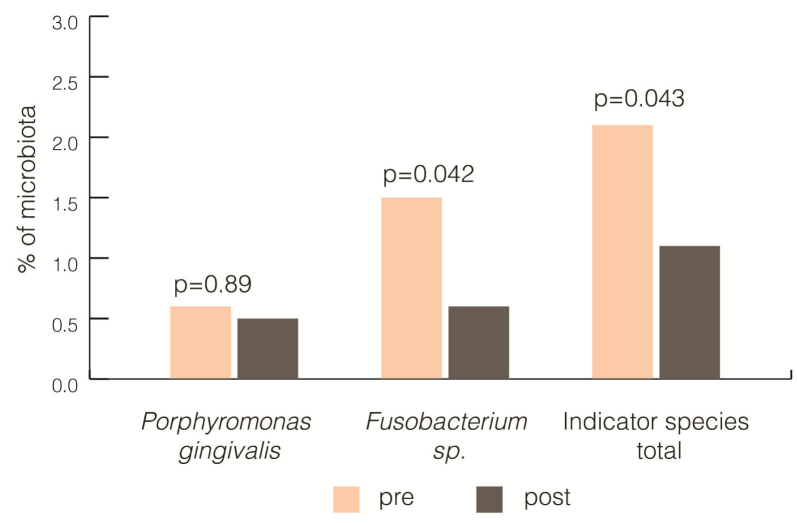

Figure 3. PetGuide treatment for four weeks in Peruvian hairless dogs showed a significant decrease in Fusobacterium sp. and infectious bacteria overall. $P$. gingivalis levels were low already before the intervention in this $\operatorname{dog}$ group $(n=5)$.

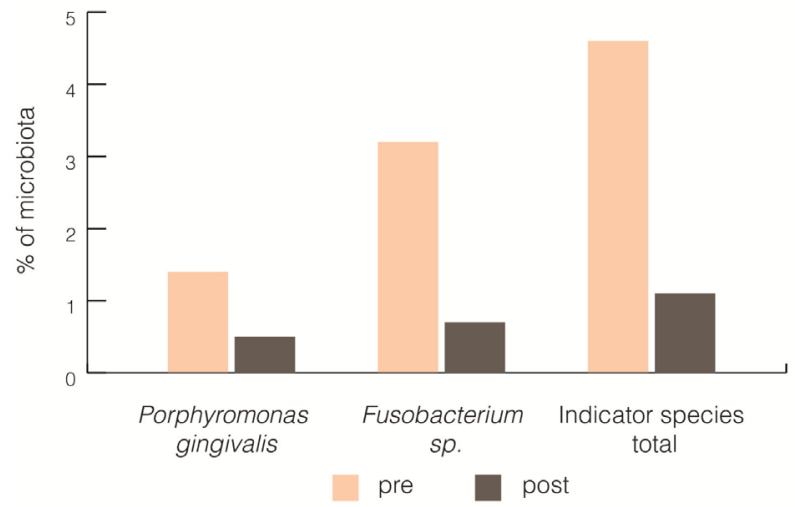

Figure 4. PetGuide treatment for four weeks with one individual Peruvian hairless dog with bad mouth/teeth health. The numbers of indicator strains were much higher than detected in other dogs. The reduction of $P$. gingivalis and Fusobacterium sp. is notable.

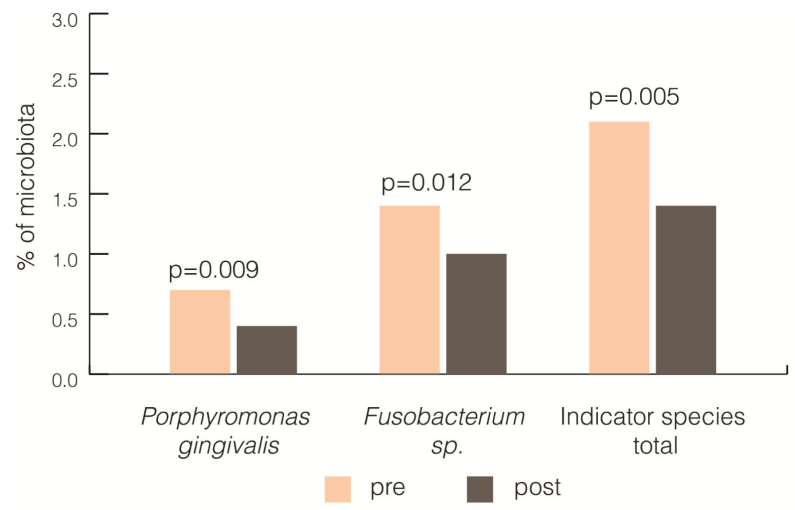

Figure 5. The treatment with PetGuide powder for four weeks reduced the amount of total gum infectious microbes significantly $(\mathrm{p}=0.005)$. Summarised results of cats and dogs $(n=22)$.

more severe diseases already at an earlier age. In this study, the PetGuide LAB powder including selected probiotic lactic acid bacteria effectively reduced the relative abundance of the infectious microbes causing the damage in teeth. 
The microbes in plaque represent numerous species with high numbers (about $10^{11} \mathrm{cfu} / \mathrm{g}$ ) in plaque, and it has been revealed that several periodontopathic species could be transmitted between humans and their companion dogs [8]. The indicator strains used in this study, P. gingivalis and Fusobacterium sp., were selected based on human trials. Since they were found in abundant levels in dogs and cats, they were used as indicators.

The dental plaque will turn into calculus which is more difficult to remove. Calculus formation and the deterioration of the oral cavity are initially caused by specific antigens or enzymes produced by plaque microbes. These compounds activate the host inflammatory response, which causes the ligaments and bones supporting the tooth to become inflamed and progressively destroyed [21]. Therefore the preventive care before any symptoms are visible is crucial. Instead of the removal of plaque, the potential pathogenic microbes causing periodontal disease can be prevented and replaced by a beneficial lactic acid bacteria (LAB) combination with the ability to adhere and colonise the gums. For domestic care, the method has to be easy for the owner and the pet.

Only four weeks of PetGuide LAB powder use significantly reduced the relative abundance of both $P$. gingivalis and Fusobacterium sp. even when the number of tested pets was low and the probiotic bacteria dose per day per pet was not accurate and even during the intervention period. A decrease in bad breath in the dogs was also reported by the owners. Using LAB powder either sprinkled on feed or during tooth brushing is a pet-friendly, easy and simple way to replace the unwanted bacteria species and decrease the risk of periodontal damages in pets.

\section{Acknowledgements}

Dr. Eveliina Munukka acts as scientific advisor of Gutguide Ltd.

\section{Conflicts of Interest}

The authors declare no conflicts of interest regarding the publication of this paper.

\section{References}

[1] Marretta, S. (2010) An Update on Periodontal Disease in Dogs. http://veterinarycalendar.dvm360.com/update-periodontal-disease-dogs-proceedin gs

[2] Niemiec, B.A. (2008) Periodontal Disease. Topics in Companion Animal Medicine, 23, 72-80. https://doi.org/10.1053/j.tcam.2008.02.003

[3] Dewirst, F.E., Klein, E.A., Thompson, E.C., Blanton, J.M., Chen, T., Milella, L., et al. (2012) The Canine Oral Microbiome. PLoS ONE, 7, e36067. https://doi.org/10.1371/journal.pone.0036067

[4] Marshall, M.D., Wallis, C.V., Milella, L., Colyer, A., Tweedie, A.D. and Harris, S. (2014) A Longitudinal Assessment of Periodontal Disease in 52 Miniature Schnauzers. BMC Veterinary Research, 10, 166.

https://doi.org/10.1186/1746-6148-10-166 
[5] Harvey, C.E., Shofer, F.S. and Laster, I. (1994) Association of Age and Body Weight with Periodontal Disease in North American Dogs. Journal of Veterinary Dentistry, 11, 94-105.

[6] Wiggs, R.B. and Lobprise, H.B. (1997) Periodontology. In: Veterinary Dentistry, Principles and Practice, Lippincott Raven Publishers, Philadelphia, PA, 186-231.

[7] Elliot, D.R., Wilson, M., Buckley, C.M. and Spratt, D.A. (2005) Cultivable Oral Microbiota of Domestic Dogs. Journal of Clinical Microbiology, 43, 5470-5476. https://doi.org/10.1128/JCM.43.11.5470-5476.2005

[8] Yamasaki, Y., Nomura, R., Nakano, K., Naka, S., Matsumoto-Nakano, M., Asai, F., et al. (2012) Distribution of Periodontopathic Bacterial Species in Dogs and Their Owners. Archives of Oral Biology, 57, 1183-1188. https://doi.org/10.1016/j.archoralbio.2012.02.015

[9] Preshaw, P.M., Seymour, R.A. and Heasman, P.A. (2004) Current Concepts in Periodontal Pathogenesis. Dental Update, 31, 570-578. https://doi.org/10.12968/denu.2004.31.10.570

[10] Kato, Y., Shirai, M., Murakami, M., Mizusawa, T., Hagimoto, A., Wada, K., et al. (2011) Molecular Detection of Human Periodontal Pathogens in Oral Swab Speciments from Dogs in Japan. Journal of Veterinary Dentistry, 28, 84-89. https://doi.org/10.1177/089875641102800204

[11] Booij-Vrieling, H.E., van der Reijden, W.A., Houwers, D.J., de Wit, W.E., Bosch-Tijhof, C.J. and Penning, L.C. (2010) Comparison of Periodontal Pathogens between Cats and Their Owners. Veterinary Microbiology, 144, 147-152. https://doi.org/10.1016/j.vetmic.2009.12.046

[12] DuPont, G.A. (1998) Prevention of Periodontal Disease. Veterinary Clinics of North America: Small Animal Practice, 28, 1129-1145. https://doi.org/10.1016/S0195-5616(98)50106-4

[13] Tenovuo, J. (2002) Clinical Applications of Antimicrobial Host Proteins Lactoperoxidase, Lysozyme and Lactoferrin in Xerostomia: Efficacy and Safety. Oral Diseases, 8, 23-29. https://doi.org/10.1034/j.1601-0825.2002.1o781.x

[14] Horz, H.-P., Meinelt, A., Houben, B. and Conrads, G. (2007) Distribution and Persistence of Probiotic Streptococcus salivarius K12 in the Human Oral Cavity as Determined by Real-Time Quantitative Polymerase Chain Reaction. Oral Microbiology Immunology, 22, 126-130. https://doi.org/10.1111/j.1399-302X.2007.00334.X

[15] Scariya, L., Nagarathna, D.V. and Varghese, M. (2015) Probiotics in Periodontal Therapy. International Journal of Pharma and Bio Sciences, 6, 242-250.

[16] Burton, J.P., Drummond, B.K., Chilcott, C.N., Tagg, J.R., Thomson, W.M., Hale, J.D.F. and Wescombe, P.A. (2013) Influence of the Probiotic Streptococcus salivarius Strain M18 on Indices of Dental Health in Children: A Randomized Double-Blind, Placebo-Controlled Trial. Journal of Medical Microbiology, 62, 875-884. https://doi.org/10.1099/jmm.0.056663-0

[17] Hatakka, K., Ahola, A.J., Yli-Knuuttila, H., Richardson, M., Poussa, T., Meurman, J.H. and Korpela R. (2007) Probiotics Reduce the Prevalence of Oral Candida in the Elderly-A Randomized Controlled Trial. Journal of Dental Research, 82, 125-130. https://doi.org/10.1177/154405910708600204

[18] Dominy, S.S., Lynch, C., Ermini, F., Benedyk, M., Marczyk, A., Konradi, A., et al. (2019) Porphyromonas gingivalis in Alzheimer's Disease Brains: Evidence for Disease Causation and Treatment with Small-Molecule Inhibitors. Science Advances, 5, eaau3333. https://doi.org/10.1126/sciadv.aau3333 
[19] Munukka, E., Leppäranta, O., Korkeamäki, M., Vaahtio, M., Peltola, T., et al. (2008) Bactericidal Effects of Bioactive Glasses on Clinically Important Aerobic Bacteria. Journal of Materials Science: Materials in Medicine, 19, 27-32. https://doi.org/10.1007/s10856-007-3143-1

[20] Vaahtovuo, J., Korkeamäki, M., Munukka, E., Viljanen, M.K. and Toivanen, P. (2005) Quantification of Bacteria in Human Feces Using 16S rRNA-Hybridization, DNA-Staining and Flow Cytometry. Journal of Microbiological Methods, 63, 276-286. https://doi.org/10.1016/j.mimet.2005.03.017

[21] Williams, R.C. (1990) Periodontal Disease. The New England Journal of Medicine, 322, 373-382. https://doi.org/10.1056/NEJM199002083220606 